CERN-AB-2003-026 (ABP)

CLIC Note 582

\title{
Main Linac Emittance Growth and Luminosity in Future Linear Colliders
}

\author{
D. Schulte
}

\begin{abstract}
Wakefield and dispersive effects in the main linac of a future linear collider can strongly affect the beam-beam interaction at the collision point [1]. For a static situation, this paper investigates the luminosity for realistic bunches in the case of the TESLA, NLC and CLIC projects assuming different degrees of collision optimisation. A part of this work was performed for the International Linear Collider Technical Review Committee [2].
\end{abstract}




\title{
MAIN LINAC EMITTANCE GROWTH AND LUMINOSITY IN FUTURE LINEAR COLLIDERS
}

\author{
D. Schulte, CERN, Geneva, Switzerland
}

\begin{abstract}
Wakefield and dispersive effects in the main linac of a future linear collider can strongly affect the beam-beam interaction at the collision point [1]. For a static situation, this paper investigates the luminosity for realistic bunches in the case of the TESLA, NLC and CLIC projects assuming different degrees of collision optimisation. A part of this work was performed for the International Linear Collider Technical Review Committee [2].
\end{abstract}

\section{INTRODUCTION}

Future linear colliders require very small beam sizes at the interaction point (IP) in order to achieve high luminosity. The high density of each beam at collision leads to the creation of a strong electro-magnetic field which focuses the oncoming beam. The beam-beam interaction is strong enough to actually change the beam size during the collision, which results in an increase of the luminosity by a factor of typically $1.5-2$ compared to the case without beambeam forces. However, the deflection of the beam particles leads to the emission of beamstrahlung, which is similar to synchrotron radiation. Many of the electron-positron collisions will thus take place with a centre-of-mass energy lower than the nominal value. To minimise this effect but still obtain high luminosity flat beams are used in which the horizontal RMS beam size $\sigma_{x}$ is much larger than the vertical $\sigma_{y}$, see the parameters in Table 1 .

The strength of the beam-beam interaction can be conveniently described using the disruption parameters $D_{x, y}$ :

$$
D_{x, y}=\frac{2 N r_{e} \sigma_{z}}{\gamma \sigma_{x, y}\left(\sigma_{x}+\sigma_{y}\right)}
$$

Here, $N$ is the number of particles per bunch, $\sigma_{z}$ the RMS bunch length and $r_{e}$ the classical electron radius. For large $D$ the beam-beam effects are important while for $D \ll 1$ the each beam acts as a thin lens. In the proposed machines one finds $D_{x} \ll 1$ and $D_{y} \gg 1$, so we will concentrate on the vertical plane.

For large $D$, it has been found that taking into account the full 6-dimensional correlation of the beam distribution for the simulation of the beam-beam collision is very important [1]. In the case of TESLA (with an older parameter set) a very small emittance increase of about $1 \%$ could lead to $20 \%$ luminosity reduction, if the beam-beam collision is not optimised.

In the following, the possibility to restore the luminosity by optimising collision offset and angle of the two beams is investigated. Also the effect on the beamstrahlung is considered. Since in many physics experiments only the fraction of the luminosity with a centre-of-mass energy close to the nominal value $E_{\mathrm{cm}, 0}$ is of interest, the luminosity $\mathcal{L}_{\text {peak }}$, which contains all collisions with $\Delta E_{c m} / E_{c m, 0}<$ 0.01 is introduced as a figure of merit. Of particular interest are TESLA because of its high disruption parameter and CLIC at $E_{c m}=3 \mathrm{TeV}$ because in this machine the beambeam effect is so strong that many particles loose the major part of their energy through beamstrahlung and hence experience a much larger effective disruption than indicated in table 1.

\section{SIMULATION PROCEDURE}

All projects foresee a certain budget for the emittance growth due to imperfections from the damping ring to the IP (from the beginning of the linac to its end, in case of CLIC). As a simplification, it is assumed in the following that all emittance growth is due to the main linac. Relatively complex beam-based alignment techniques are foreseen to minimise this effect. In the simulation we replace these techniques by a simple one-to-one steering and scale the imperfections such that we achieve an aver-

Table 1: Some beam parameters at the interaction point of the different machines. In the case of CLIC the transverse sizes are obtained by fits, since the beam distribution is not very Gaussian. The beam consists of trains of $n_{b}$ bunches, which are delivered with a repetition frequency $f_{r}$. In case of NLC and CLIC the luminosities $\mathcal{L}_{0}$ differ slightly from those in [2] because the latter allow for some other effects. The disruption parameters $D_{x}$ and $D_{y}$ are calculated using the nominal emittance at the IP, these are in case that the emittance growth is completely uncorrelated.

\begin{tabular}{|c|c|c|c|c|c|}
\hline & unit & TESLA & NLC & CLIC & CLIC \\
\hline$N$ & {$\left[10^{9}\right]$} & 20 & 7.5 & 4 & 4 \\
$\sigma_{z}$ & {$[\mu \mathrm{m}]$} & 300 & 110 & 35 & 35 \\
$\sigma_{x}$ & {$[\mathrm{~nm}]$} & 554 & 243 & 204 & 60 \\
$\sigma_{y}$ & {$[\mathrm{~nm}]$} & 5.0 & 3.0 & 1.2 & 0.7 \\
$E$ & {$[\mathrm{GeV}]$} & 250 & 250 & 250 & 1500 \\
\hline$f_{r}$ & {$[\mathrm{~Hz}]$} & 5 & 120 & 200 & 100 \\
$n_{b}$ & & 2820 & 195 & 154 & 154 \\
$\mathcal{L}_{0}$ & {$\left[10^{34} \mathrm{~m}^{-2} \mathrm{~s}^{-1}\right]$} & 3.4 & 2.1 & 2.3 & 8.0 \\
$P_{b}$ & $\mathrm{MW}$ & 11.3 & 7.0 & 4.9 & 7.4 \\
\hline$D_{x}$ & & 0.22 & 0.16 & 0.04 & 0.07 \\
$D_{y}$ & & 24.8 & 12.8 & 6.6 & 6.3 \\
\hline
\end{tabular}


Table 2: The emittances used (initial and final in the vertical plane) and the misalignments used to mimic the static errors of the machines. After application of the errors only a one-to-one correction was performed. If more sophisticated beam-based alignment schemes were used, much larger errors would be permitted.

\begin{tabular}{|c|c|c|c|c|c|}
\hline & unit & TESLA & NLC & CLIC & CLIC \\
\hline$\epsilon_{x}$ & {$[\mu \mathrm{m}]$} & 10 & 3.6 & 2 & 0.68 \\
$\epsilon_{y, i} / \epsilon_{y, f}$ & {$[\mathrm{~nm}]$} & $20 / 30$ & $20 / 40$ & $5 / 10$ & $5 / 10$ \\
\hline$\sigma_{B P M}$ & {$[\mu \mathrm{m}]$} & 25 & 5.0 & 0.72 & 0.4 \\
$\sigma_{c a v}$ & {$[\mu \mathrm{m}]$} & 500 & 12.5 & 8.0 & 4.5 \\
$\sigma_{c a v}^{\prime}$ & {$[\mu \mathrm{rad}]$} & 300 & 100 & 8.0 & 4.5 \\
\hline
\end{tabular}

Table 3: Luminosities found for all machines, before correction $\left(\mathcal{L}_{1}\right)$, after offset optimisation $\left(\mathcal{L}_{o f f}\right)$ and after further angle optmisation $\left(\mathcal{L}_{\text {angle }}\right)$.

\begin{tabular}{|c|c|c|c|c|c|}
\hline & unit & TESLA & NLC & CLIC & CLIC \\
\hline $\mathcal{L}_{0}$ & {$\left[10^{34} \mathrm{~m}^{-2} \mathrm{~s}^{-1}\right]$} & 3.4 & 2.1 & 2.3 & 8.0 \\
\hline $\mathcal{L}_{1}$ & {$\left[10^{34} \mathrm{~m}^{-2} \mathrm{~s}^{-1}\right]$} & 2.2 & 1.8 & 2.2 & 7.4 \\
$\mathcal{L}_{\text {off }}$ & {$\left[10^{34} \mathrm{~m}^{-2} \mathrm{~s}^{-1}\right]$} & 2.5 & 2.1 & 2.5 & 7.9 \\
$\mathcal{L}_{\text {ang }}$ & {$\left[10^{34} \mathrm{~m}^{-2} \mathrm{~s}^{-1}\right]$} & 3.3 & 2.2 & 2.6 & 8.1 \\
\hline
\end{tabular}

age emittance growth equivalent to the budget. The relative sizes of the imperfections - misalignment of the beamposition monitors (BPMs) and the structures and tilt of the structures-are chosen to represent the performance of the more complex alignment algorithm. Table 2 lists the initial and final emittances and the alignment errors used. It should be noted that the emittance varies from machine to machine and that only the average of the machines simulated is consistent with the budget.

The emittance growth in the main linac is simulated using PLACET [3]. Then the mean beam angle and offset are corrected before the beam is transported through the beam delivery system, also using PLACET. A number of machines with different seeds for the random number generators are simulated and their angle and offset at the IP are corrected. Pairs of these beams are fed into the beam-beam simulation code GUINEA-PIG [4].

In the optimisation, first the relative offset of the two colliding beam is varied as to maximise luminosity. Then the vertical crossing angle is modified, this certainly can change the projected emittance. Several options exist to achieve such a modification; here we just modify position and crossing angle at the IP without worrying about the actual implementation.

Since the beam-beam collision is not very stable, in particular in the case of TESLA, the convergence of the beambeam simulation has to be carefully checked, details can be found in [5].

\section{AVERAGE TOTAL LUMINOSITY}

The luminosities found in the simulation are compared to the target values in table 3 . In case of NLC and CLIC

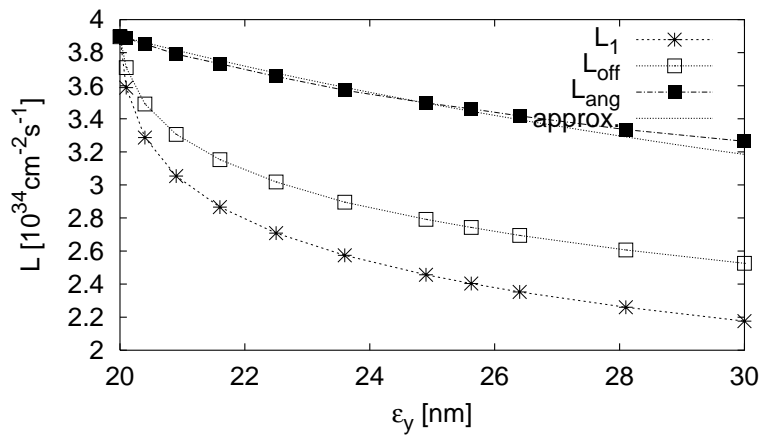

Figure 1: The luminosity as a function of the emittance at the end of the linac in the case of TESLA. For comparison, the expected luminosity using the simple scaling $\mathcal{L} \propto \sqrt{\epsilon_{y}}$ is also shown.

the achieved values are not far from the goal even without performing an offset and angle optimisation. In the case of TESLA the optimisation is essential. In all cases the achieved luminosity is consistent with the design value. A second iteration of the very simplistic optimisation procedure leads to a very small further improvement. The two parameters offset and angle seem thus quite orthogonal.

In TESLA it was foreseen to longitudinally move the beam waist slightly before the collision point, since this yields higher luminosity [6]. A further optimisation was therefore attempted for TESLA. First the waists of both beams were longitudinally moved in a symmetric fashion, then in an asymmetric way. The improvement was tiny, about $2 \%$, without correlation it raised the luminosity by about $15 \%$ from $3.0 \times 10^{34} \mathrm{~cm}^{-2} \mathrm{~s}^{-1}$ to $3.4 \times$ $10^{34} \mathrm{~cm}^{-2} \mathrm{~s}^{-1}$ It remains to be investigated if a better procedure could yield better performance.

One can conclude that all projects can on average about achieve the target luminosity if the design emittance goal can be met. Within limits, the emittance can serve as a reasonable measure to predict the luminosity after performing the optimisation. This is exemplified is Fig. 1, which shows the luminosity in the most interesting case of TESLA as a function of the emittance at the IP. Before optimisation, a small growth leads to large reduction in luminosity. After full optimisation the luminosity seems to scale as $\mathcal{L} \propto{\sqrt{\epsilon_{y}}}^{1}$.

\section{MACHINE-TO-MACHINE VARIATIONS}

The luminosity of an individual beam-beam collision can be predicted from the emittances of the two beams with a limited precision. The dependence $\mathcal{L} \propto 1 / \sqrt{\epsilon_{y, 1}+\epsilon_{y, 2}}$ is only approximately valid, see Fig. 2 for TESLA. Here the actual distribution needs to be taken into account.

\footnotetext{
${ }^{1}$ It should be noted that for larger variations of the vertical emittance the beam-beam interaction is also affected and the simple $\mathcal{L} \propto \sqrt{\epsilon_{y}}$ does not hold true, this is indepenent of whether the correlations are taken into account or not.
} 


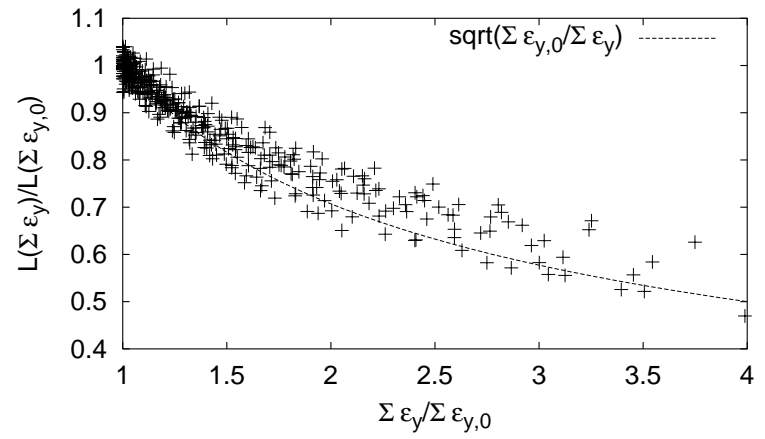

Figure 2: The luminosity of the individual collisions as a function of the sum of the two vertical emittances (TESLA).
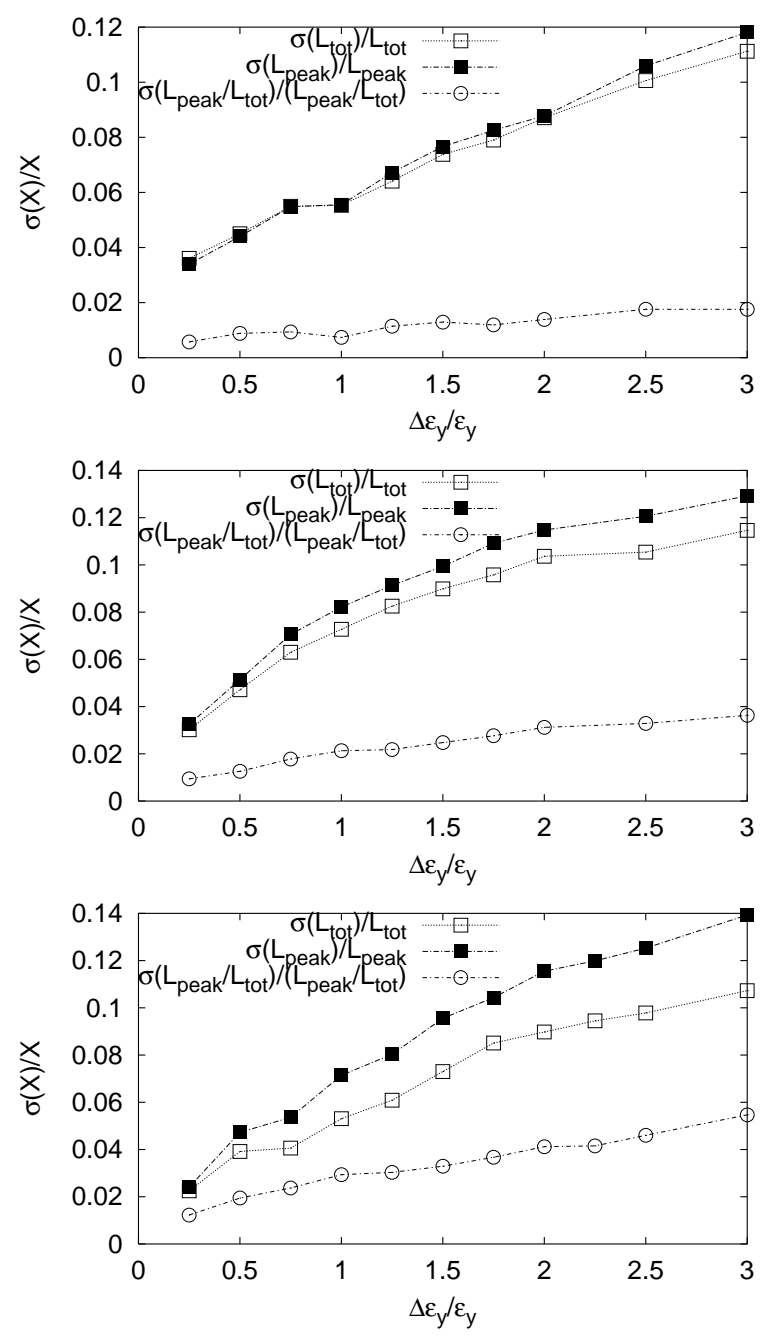

Figure 3: RMS-fluctuations of the luminosity as a function of the emittance growth in the main linac for TESLA, NLC and CLIC (at $E_{c m}=3 \mathrm{TeV}$ ), respectively.
The realistic beam distributions not only affects the total luminosity but also the amount of beamstrahlung radiated by the beams and thus the luminosity spectrum. If two beams collide with a small vertical offset they emit more beamstrahlung. In the case of realistic beams, fractions of them will actually collide with an offset which might yield more beamstrahlung and affect the luminosity $\mathcal{L}_{\text {peak }}$ close to the nominal centre-of-mass energy.

Above, the alignment errors in the main linac were chosen to yield an average emittance growth equal to the budget. In the following, the sizes of these errors are determined for each individual machine to yield exactly the target emittance. This allows to determine the RMS fluctuation of the luminosity for machines with the same emittance.

For the average total luminosity the results are similar to the previous ones, the emittance budgets are consistent with the target luminosities. The change of the ratio $\mathcal{L}_{\text {peak }} / \mathcal{L}_{\text {tot }}$ due to the emittance growth is small, less than $1 \%$ in all machines. But, as can be seen in Fig. 3, the RMS luminosity variations of the individual machines increases with the emittance growth; in the case of CLIC at $E_{c m}=3 \mathrm{TeV}$ the beamstrahlung adds significantly to the variations of $\mathcal{L}_{\text {peak }}$.

\section{CONCLUSION}

In the strong beam-beam interaction regime a very small emittance increase can lead to a large luminosity reduction. It has been shown that this strong reduction can be cured by optimising the collision offset and angle. This requires a luminosity monitor and sufficiently stable beam conditions. In this case one can first optimise the collision offset and then the angle to recover most of the lost luminosity. Dynamic imperfections may be too fast to allow the use of a luminosity monitor and can therefore stay problematic, see also [7]. After optimisation, the ratio of the luminosity close the nominal centre-of-mass energy to the total one $\mathcal{L}_{\text {peak }} / \mathcal{L}_{\text {tot }}$ does on average not depend strongly on the correlated emittance growth, but the RMS fluctuation from machine to machine do.

\section{REFERENCES}

[1] R. Brinkmann, O. Napoly and D. Schulte. CLIC-Note 505 (2001).

[2] The International Linear Collider Technical Review Committee is headed by G. Loew. SLAC-R-606 (2003).

[3] E. D'Amico, G. Guignard, N. Leros, D. Schulte. CERN/PS 2001-028 (AE).

[4] D. Schulte. CERN/PS 99-014 (LP).

[5] D. Schulte. CLIC-Note 544 (2002).

[6] D. Schulte. PhD. thesis. TESLA 97-08 (1996).

[7] A. Seryi et al. This conference. 